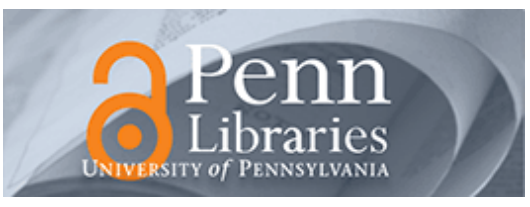

University of Pennsylvania

ScholarlyCommons

$9-2006$

\title{
Prone Positioning can be Safely Performed in Critically III Infants and Children
}

Lori D. Fineman

Michelle A. LaBrecque

Mei-Chiung Shih

Martha A. Q. Curley

University of Pennsylvania, curley@nursing.upenn.edu

Follow this and additional works at: https://repository.upenn.edu/nrs

Part of the Critical Care Nursing Commons, and the Pediatric Nursing Commons

\section{Recommended Citation}

Fineman, L. D., LaBrecque, M. A., Shih, M., \& Curley, M. A. (2006). Prone Positioning can be Safely Performed in Critically III Infants and Children. Pediatric Critical Care Medicine, 7(5), 413-422.

http://dx.doi.org/10.1097/01.PCC.0000235263.86365.B3

This is a non-final version of an article published in final form in Pediatric Critical Care Medicine. 2006 September ; 7(5): 413-422. doi:10.1097/01.PCC.0000235263.86365.B3.

This paper is posted at ScholarlyCommons. https://repository.upenn.edu/nrs/17

For more information, please contact repository@pobox.upenn.edu. 


\title{
Prone Positioning can be Safely Performed in Critically III Infants and Children
}

\author{
Abstract \\ Objective: To describe the effects of prone positioning on airway management, mechanical ventilation, \\ enteral nutrition, pain and sedation management, and staff utilization in infants and children with acute \\ lung injury.
}

Design: Secondary analysis of data collected in a multiple-center, randomized, controlled clinical trial of supine vs. prone positioning.

Setting: Seven pediatric intensive care units located in the United States.

Patients: One hundred and two pediatric patients (51 prone and 51 supine) with acute lung injury.

Interventions: Patients randomized to the supine group remained supine. Patients randomized to the prone group were positioned prone per protocol during the acute phase of their illness for a maximum of 7 days. Both groups were managed using ventilator and sedation protocols and nutrition and skin care guidelines.

Measurements and Main Results: Airway management and mechanical ventilatory variables before and after repositioning, enteral nutrition management, pain and sedation management, staff utilization, and adverse event data were collected for up to 28 days after enrollment. There were a total of 202 supineprone-supine cycles. There were no differences in the incidence of endotracheal tube leak between the two groups $(p=.30)$. Per protocol, $95 \%$ of patients remained connected to the ventilator during repositioning. The inadvertent extubation rate was 0.85 for the prone group and 1.03 for the supine group per 100 ventilator days $(p=1.00)$. There were no significant differences in the initiation of trophic $(p=$ $.24)$, advancing $(p=.82)$, or full enteral feeds $(p=.80)$ between the prone and supine groups; in the average pain $(p=.81)$ and sedation $(p=.18)$ scores during the acute phase; and in the amount of comfort medications received between the two groups $(p=.91)$. There were no critical events during a turn procedure. While prone, two patients experienced an obstructed endotracheal tube. One patient, supported on high-frequency oscillatory ventilation, experienced persistent hypercapnea when prone and was withdrawn from the study. The occurrence of pressure ulcers was similar between the two groups ( $p$ $=.71)$. Compared with the supine group, more staff $(p \leq .001)$ and more time were necessary to reposition patients in the prone group.

Conclusions: Our data show that prone positioning can be safely performed in critically ill pediatric patients and that these patients can be safely managed while in the prone position for prolonged periods of time.

\section{Keywords}

prone position, airway, mechanical ventilation, enteral nutrition, pain, sedation, pressure ulcer, resource utilization, multiple-site study, acute lung injury, adult respiratory distress syndrome

\section{Disciplines}

Critical Care Nursing | Medicine and Health Sciences | Nursing | Pediatric Nursing

\section{Comments}

This is a non-final version of an article published in final form in Pediatric Critical Care Medicine. 2006 September ; 7(5): 413-422. doi:10.1097/01.PCC.0000235263.86365.B3. 
Published in final edited form as:

Pediatr Crit Care Med. 2006 September ; 7(5): 413-422.

\title{
Prone Positioning can be Safely Performed in Critically III Infants and Children
}

\author{
Lori D. Fineman, R.N., M.S. ${ }^{1}$, Michelle A. LaBrecque, R.N., M.S.N. ${ }^{2}$, Mei-Chiung Shih, Ph.D. \\ , and Martha A.Q. Curley, R.N., Ph.D. ${ }^{2}$ \\ 1 Pediatric Cardiac Intensive Care, University of California San Francisco Children's Hospital; \\ 2 Critical Care and Cardiovascular Nursing, Childrens Hospital Boston; \\ 3 Clinical Research Program, Children's Hospital Boston.
}

\begin{abstract}
Objective- To describe the effects of prone positioning on airway management, mechanical ventilation, enteral nutrition, pain and sedation management and staff utilization in infants and children with acute lung injury (ALI).
\end{abstract}

Design-Secondary analysis of data collected in a multi-center, randomized, controlled clinical trial of supine versus prone positioning.

Patients and Setting - 102 pediatric patients (51 prone and 51 supine) with ALI from seven pediatric intensive care units located in the Untied States.

Interventions- Patients randomized to the supine group remained supine. Patients randomized to the prone group were positioned prone per protocol during the acute phase of their illness for a maximum of 7 days. Both groups were managed using ventilator and sedation protocols and nutrition and skin care guidelines.

Measurements- Airway management and mechanical ventilatory parameters pre and post repositioning, enteral nutrition management, pain and sedation management, staff utilization, and adverse event data collected for up to 28 days after enrollment.

Main Results - There were a total of 202 supine-prone-supine cycles. There were no differences in the incidence of endotracheal tube leak between the two groups $(p=0.30)$. Per protocol, $95 \%$ of patients remained connected to the ventilator during repositioning. The inadvertent extubation rate was 0.85 for the prone group and 1.03 for the supine group per 100 ventilator days $(p=1.00)$. There were no significant differences in the initiation of trophic $(p=0.24)$, advancing $(p=0.82)$ or full enteral feeds $(p=0.80)$ between the prone and supine groups and in the average pain $(p=0.81)$ and sedation $(p=0.18)$ scores over the acute phase and in the amount of comfort medications received between the two groups. ( $p=.91)$. There were no critical events during a turn procedure. While prone, two patients experienced an obstructed ETT. One patient, supported on HFOV, experienced persistent hypercapnea when prone and was withdrawn from the study. The occurrence of pressure ulcers was similar between the two groups $(p=0.71)$. Compared to the supine group, more staff $(p \leq 0.001)$ and more time were necessary to reposition patients in the prone group.

Corresponding Author: Martha A.Q. Curley, RN, PhD; Children's Hospital, Boston; Farely 559; 300 Longwood Ave. Boston, MA 02115; Office:617-355-6886; Fax: 617-730-0126; Martha.Curley@ Childrens.Harvard.edu.

"The authors have not disclosed any potential conflicts of interest."

Funding: Funding Support: NIH/NINR RO1NR05336; Equipment support: Novametrix Medical Systems, Medical Ventures, i-STAT Corporation. 
Conclusions- Our data show that prone positioning can be safely performed in critically ill pediatric patients and that these patients can be safely managed while in the prone position for prolonged periods of time.

\section{Keywords}

Prone; position; airway; mechanical ventilation; enteral nutrition; pain; sedation; pressure ulcer; resource utilization; multi-site study; acute lung injury; Adult Respiratory Distress Syndrome (ARDS)

Prone positioning is a therapeutic maneuver that improves oxygenation in pediatric and adult patients with acute lung injury (ALI) supported on mechanical ventilation.(1-7) Although the short-term effect of prone positioning on oxygenation is well described, clinical trials in both adult and pediatric patients have demonstrated that prone positioning does not improve a patient's clinical outcome, specifically, patient survival, ventilator-free days, or recovery from lung injury.(8-11)

Without evidence of a positive impact on patient outcomes, prone positioning should not be used in the routine management of pediatric patients with ALI. However, intermittent prone positioning may be helpful as a recruitment maneuver to improve oxygenation in the hypoxic patient with ALI. Prone positioning is also occasionally used in the pediatric critical care population without ALI to optimize patient comfort, manage complex wounds, and prevent pressure ulcers. Therefore, a safe and reliable method for prone positioning critically ill pediatric patients is important so that potential complications associated with the prone position can be avoided. Reported complications include episodes of hemodynamic instability, desaturation and supraventricular tachycardia; inadvertent endotracheal tube (ETT) extubation and/or obstruction; removal of essential tubes and vascular catheters; pressure ulcers; and enteral feeding intolerance. (12) Also important is a comprehensive description of the effect of prone positioning on clinically important parameters in the management of the critically ill pediatric patient. The purpose of this paper is to describe the effects of prone positioning on airway management, mechanical ventilatory requirements, enteral nutrition management, pain and sedation management, and staff utilization in critically ill infants and children.

\section{METHODS}

The study design has been previously described in detail.(9) In brief, patients with ALI were recruited from seven Pediatric Intensive Care Units located in the USA that participate in the Pediatric Acute Lung Injury and Sepsis Investigators (PALISI) Network. One hundred and two patients were enrolled from August 2001 through April 2004. The institutional review board of each hospital approved the study. Written informed consent was obtained from the parent or legal guardian of each subject.

Inclusion criteria were pediatric patients ( 2 weeks to 18 years) who were intubated and mechanically ventilated with a $\mathrm{PaO}_{2}: \mathrm{FiO}_{2}$ ratio of 300 or less, bilateral pulmonary infiltrates, and no clinical evidence of left atrial hypertension.(13) Patients were excluded if they were less than 2 weeks of age (newborn physiology), less than 42 weeks post-conceptual age (considered preterm), unable to tolerate a position change (persistent hypotension, cerebral hypertension); had respiratory failure from cardiac disease; had hypoxemia without bilateral infiltrates; had received a bone marrow or lung transplant; were supported on extracorporeal membrane oxygenation; had a nonpulmonary condition that could be exacerbated by the prone position; had participated in other clinical trials within the preceding 30 days; or if there was a decision to limit life support. Randomization was done using a permuted blocks design, 
stratified by center, with random block sizes. Allocation was concealed; each center received serially numbered, opaque, sealed envelopes containing study assignments.

\section{Positioning Protocol (see Appendix)}

Prone positioning was accomplished by protocol, which included a procedural checklist, developed during our Phase One study. (5) All patients were maintained on standard hospital beds. Individually sized head, chest, pelvic, distal femoral, and lower limb cushions were created using pressure relieving material (covered Eggcrate; Span American Medical Systems, Greenville, SC; or Bendy ${ }^{\circledR}$ Bumper (foam only); Medical Ventures; Weymouth, MA). When properly positioned the patient's body folded into the cushions, the abdomen was not compressed and the shoulders not hyperextended.

Prior to prone positioning, the security of all invasive devices was reassessed and ETT position was checked on the most recent chest radiograph. The tip of the ETT was positioned deeper than one third of the total length of the thoracic trachea before prone positioning to prevent it from moving into the cervical trachea when prone (23). The respiratory therapist checked for the presence of an ETT leak and, if necessary, inflated the ETT cuff using minimal leak technique.

The turn protocol recommended that the bedside nurse- respiratory team coordinate the turn with the assistance of one or two additional assistants. During the turn procedure, the respiratory therapist was assigned the primary responsibility of ETT protection. Infants/ toddlers were lifted up, turned 45 degrees, turned prone on their cushions. School aged and adolescent patients were turned using the mummy technique. During each turn the patient's head was kept in alignment with the body, avoiding hyperextension.

Patients were turned toward the ventilator without disconnecting from the ventilator. If the patient required ETT suctioning, turning was delayed until the patient was suctioned and returned to their pre-suctioning ventilator settings. If the patient was supported on high frequency oscillatory ventilation (HFOV) the care team was cautioned to reassess the patient's chest wiggle immediately after prone positioning.

Prone positioning included a 2-hour cyclic rotation from full prone to right lateral/prone to full prone to left lateral/prone then to full prone. When prone, the patient's head pillow was positioned to prevent extreme lateral head rotation and the patient's upper arms remained at their side and their lower arms were flexed up. The lower limbs were cushioned so that the patient's knees and toes were positioned off the bed. When tilted into a lateral prone position, the patient's dependent lower arm was repositioned against their torso and their non-dependent lower arm was flexed at the elbow and positioned up towards the patient's head. Ten hours into the prone position the patient's upper chest and head were lifted so that their head could be turned in the opposite direction.

Prone positioning continued each day during the acute phase of their ALI illness for a maximum of 7 days of treatment. The acute phase was defined as the time interval between randomization and the time at which the patient met criteria for extubation readiness testing: specifically, spontaneous breathing, oxygenation index (mean airway pressure/[ $\mathrm{PaO}_{2}: \mathrm{FiO}_{2}$ ratio] x 100) less than 6 , and a decrease or plateau in ventilator support over the previous 12 hours.(14) Extubation readiness testing criteria were assessed in both groups each morning when supine. If criteria were met the acute phase ended and patients were tested for extubation readiness. 


\section{Non-positional Protocols}

The ventilator protocol used a low tidal volume/open lung strategy (16-18) and permissive hypercapnea. $(19,20)$ During repositioning, the $\mathrm{FiO}_{2}$ could be manipulated to maintain the target $\mathrm{SpO}_{2}$ but all other ventilator settings were to be held constant until one hour after repositioning. Exhaled tidal volume (Vt), as measured at the airway using a $\mathrm{CO}_{2} \mathrm{SMO}^{+}$monitor (Novametrix Medical Systems Inc; Wallingford, CT, USA), was limited to $6 \mathrm{~mL} / \mathrm{kg}$ of predicted body weight. Although a patient could be transitioned to HFOV when their OI was 15 and rising, the use of HFOV was mandated with an OI of 20. The head of the bed was elevated 30 degrees in supine patients and placed in a 30 degree reverse trendelenburg position in prone patients. (21)

Sedation in both groups was managed by the nurse-implemented sedation protocol. (22) Essential elements included pain and sedation scoring every four hours using an ageappropriate pain instrument $(23,24)$ and the Modified Motor Activity Assessment Scale $(25$, 26 ) and the use of continuous benzodiazepine and opioid infusions.

The Nutrition Guideline identified the goal of adequate calories to sustain the metabolic needs of the patient. (27) Unless contraindicated, all patients were advanced to full transpyloric enteral nutrition. (28) The Skin Care and Pressure Ulcer Guideline identified the goal of skin integrity. A daily skin assessment was performed and recorded on each patient during the acute treatment phase. Universally accepted prevention strategies were used to prevent pressure ulcers. $(29,30)$ Pressure ulcers are staged and managed according to National Pressure Ulcer Advisory Panel recommendations. $(31,32)$

\section{Statistical Analysis}

We used Wilcoxon rank sum test or Fisher's exact test, as appropriate, to compare prone and supine groups in their baseline characteristics and outcomes that were calculated on a per subject basis, except for time to achieve enteral feeds (by log rank test) and inadvertent extubation rate (by exact binomial test for comparing rates of two Poission processes). Outcomes that were calculated for each study day were either analyzed by study day, via Wilcoxon rank sum test or Fisher's exact test, or analyzed in aggregate (assuming a common prone-to-supine difference across all acute phase days), via generalized estimating equations in order to account for potential correlations between observations from the same patient, where statistical significance was determined by Wald's test using empirical variance. For prone patients, we also evaluated the changes after supine-to-prone turn and prone-to-supine turn, via generalized estimating equations and Wald's tests for outcomes analyzed across all acute phase days and via signed rank tests for outcomes analyzed by study day. The number of staff and length of time involved in supine-to-prone and prone-to-supine turns were compared by study day using signed rank tests. All analyses were performed with SAS software (Version 9.0, SAS Institute, Cary, NC). A two-sided P-value of less than 0.05 indicated statistical significance.

\section{RESULTS}

We enrolled 102 patients (51 prone and 51 supine). Patient characteristics at enrollment are noted in Table 1. Demographic data are reported in aggregate because there were no significant differences between the prone and supine groups.

Patients remained in the acute phase of their illness for a median of 4 days (Q1-Q3, 2-7 days; $p=0.16$ prone vs. supine, Wilcoxon rank-sum test). In total, there were 439 acute phase study days (205 prone, 234 supine). Three patients in the prone group were withdrawn from all study protocols because of hemodynamic instability unrelated to study protocols, extracorporeal 
membrane oxygenation cannulation, and persistent hypercarbia in the prone position. Positional protocols were stopped in one prone patient with sickle cell disease who required frequent abdominal reassessment.

There were a total of 202 supine-prone-supine cycles in patients randomized to the prone group. Thirty-three cycles, on 22 patients, were less than 20 hours in duration. Patients were returned supine early for procedures (22 cycles; line placement, dialysis, nasal-jejeunal tube [NJT] placement, radiological studies) or for assessment and management of a change in patient condition while prone ( 9 cycles; desaturation, evolving acidosis, hypercarbia, hypotension, hypothermia). Bedside clinicians opted to forego daily prone positioning for two cycles; one for respiratory and hemodynamic instability while supine and one for increased sedation requirement in the prone position in a patient with Trisomy 21 on his last acute phase day.

\section{Airway Management}

Except for one patient with a tracheostomy in the prone group, all patients were orally intubated (ETT size 4.5 median; Q1-Q3, 3.5-6; $p=0.39$ prone vs. supine; Wilcoxon rank sum test). Most patients had cuffed endotracheal tubes (72\% prone, $64 \%$ supine, $p=0.52$ Fisher's exact test). Although more patients with an uncuffed ETT experienced an air leak (36\% uncuffed versus $24 \%$ cuffed) this difference was not statistically significant ( $p=0.07$, Wald's test).

There were no significant differences between prone and supine groups in the incidence of ETT leak (31\% prone and $24 \%$ supine; $p=0.30$, Wald's test). There was also no significant differences between the two groups in the incidence of ETT leak for either conventional mechanical ventilation (CMV: $36 \%$ prone and $30 \%$ supine, $p=0.51$, Wald's test) or HFOV ( $21 \%$ prone and $8 \%$ supine; $p=0.14$ Wald's test). The average measured air leak by CO2SMO + during the acute phase was $3.2 \%$ (prone $3.6 \%$ and supine $2.9 \%, p=0.49$ Wald's test). In prone patients, there were no significant differences in the incidence of ETT leak when positioned prone or supine ( $32 \%$ while prone, $28 \%$ while supine; $\mathrm{P}=0.06$, Wald's test). Results remained non-significant when stratified by mode of ventilation (CMV: $40 \%$ prone and $35 \%$ supine, $p=0.06$; HFOV: $16 \%$ prone and supine, $p=1.00$; Wald's test).

There were 9 inadvertent extubations during the entire study period; 5 events in 5 supine patients and 4 events in 3 prone patients. Of the 4 inadvertent extubations in the prone group, 2 occurred while the patient was supine and none occurred during repositioning. The inadvertent extubation rate was 0.85 for the prone group and 1.03 for the supine group per 100 ventilator days ( $p=1.00$; exact binomial test for comparing rates of Poisson processes; total ventilator days: supine 483 and prone 472; $p=0.73$; Wilcoxon rank sum test).

\section{Mechanical ventilation}

Per protocol, nearly all prone patients remained connected to the ventilator during repositioning (96\% supine-to-prone turn; 94\% prone-to-supine) and most ventilator settings were held constant for one hour after repositioning (see Table 2). When supported on CMV, the $\mathrm{FiO}_{2}$ was decreased during the supine-to-prone turn. When supported on HFOV the $\mathrm{FiO}_{2}$ was increased during the prone-to-supine turn. When supported on CMV, the mean airway pressure was lower after a prone-to-supine turn. If volume control mode was used, the pressure plateau was also lower after a prone-to-supine turn.

\section{Enteral nutrition}

Among survivors, trophic feeds were initiated on study day 2 (median; Q1-Q3, 1-4), advancement of enteral feeds began on study day 3 (median; Q1-Q3, 1-6) and full enteral feeds were reached by study day 5 (median; Q1-Q3, 3-10). No significant differences were noted in the initiation of trophic, advancing or full enteral feeds between the prone and supine 
groups (trophic $p=0.24$, advancing $p=0.82$ and full feeds $p=0.80, \log$ rank test). Forty-two percent of the patients achieved full enteral nutrition during the acute phase (prone $37 \%$, supine $48 \%, p=0.40$, Fisher's exact test). Among those who reached full feeding during the acute phase, $85 \%$ were fed via the jejeunal route on the first day of full feeding, while $15 \%$ were fed via the gastric route. Among those who reached full feeding during the entire 28-day study period, patients who were fed via the jejeunal route reached full feeding earlier than those fed via the gastric route (jejeunal: median day 4, Q1-Q3, 2-7.5 days; gastric: median day 7; Q1$\mathrm{Q} 3,4-12$ days; $p=0.03$, Wilcoxon rank sum test). No adverse events were reported related to enteral feedings.

\section{Pain and sedation management}

The patient's pain and sedation scores during the acute phase were low in both groups (pain: median 0; Q1-Q3, $0-0 ; p \geq 0.20$ by study day, Wilcoxon rank sum test; MMAAS: median -1 ; $\mathrm{Q} 1-\mathrm{Q} 3,-1$ to $0 ; p \geq 0.06$ by study day; Wilcoxon rank sum test). The average pain and sedation scores over the acute phase also did not differ between the two groups (pain: $p=0.81$, Wilcoxon rank sum test; MMAAS, $p=0.18$, Wilcoxon rank sum test). Compared to the supine group, significantly more patients in the prone group received a bolus of a comfort medication prior to a turn ( $47 \%$ in prone-to-supine turn in prone patients, $13 \%$ in side-to-side turn in supine patients; $p \leq 0.04$ for days $1-6 ; p=0.25$ on day 7; Fisher's exact test). However, there were no significant differences in the total sedation administered between the two groups $(p=.91)$. (9) In prone patients, there were no significant differences in either pain or sedation scores before and after repositioning (supine-to-prone: $p \geq 0.12$, prone-to-supine: $p \geq 0.25$; signed rank test).

\section{Adverse events}

There were no critical events during a turn procedure; specifically, no episodes of hemodynamic instability, persistent desaturation, dysrhythmias, or endotracheal extubation.

During the acute phase, $61 \%$ of all patients received neuromuscular blockade (68\% prone; $54 \%$ supine; $p=0.22$ Fisher's exact test). Not including low-dose dopamine ( $\leq 5 \mathrm{mcg} / \mathrm{kg} / \mathrm{min}), 50 \%$ of patients required the simultaneous administration of up to four vasopressor/inotropic medications during a 24 hour period ( $44 \%$ prone vs. 56\% supine; $p=0.32$; Fisher's exact test). No central line, infusing these agents, was dislodged or compressed during a turn procedure. Approximately $12 \%$ of patients had $1-3$ chest tubes (10\% prone, $14 \%$ supine; Fisher's exact test $p=0.76$ ). No chest tube was dislodged during a turn procedure and there were no reported critical incidents related to chest tube management.

While prone, two patients experienced an obstructed ETT, one patient with bronchiolitis with pneumonia and the other with pneumonia. Three patients in the prone group experienced five episodes of transient desaturation. Four of these episodes occurred in infants with bronchiolitis with pneumonia and the fifth occurred in a patient with pneumonia who was noted to have thick ETT secretions. Two episodes occurred when patients, randomized to the prone group, were in the supine position. Of note, two patients in the supine group (one bronchiolitic with pneumonia and the other with pneumonia) experienced six episodes of transient desaturation. One patient in the prone group also experienced several episodes of sinus bradycardia on four separate study days. The etiology of these events, which occurred with and without stimulation, was never determined by the clinical team and the episodes resolved with recovery from ALI.

In addition to the patient in the prone group who was withdrawn from all study protocols because of persistent hypercarbia while in the prone position, four additional patients randomized to the prone group experienced transient hypercarbia. At the time of the 
hypercarbic event, all patients were supported on HFOV and the events occurred when patients were in both prone and supine positions.

Eighteen patients ( 10 prone, 8 supine, $p=0.8$, Fisher's exact test) developed a total of 26 Stage II or greater pressure ulcers over the 28-day study period (13 prone, 13 supine, $p=0.71$, Wilcoxon rank sum test) (see Table 3). The worse study-related pressure ulcer, Stage III occipital, occurred in an infant in the supine group. Two patients in the prone group also developed preventable skin injuries: circumoral rash from facial contact with oral secretions and paraphimosis from an unretracted foreskin.

\section{Staff utilization}

Compared to the supine group, twice as many staff members were used to reposition patients in the prone group (4 median; Q1-Q3, 3-5 prone vs. 2 median; Q1-Q3, $1-2$ supine; $p \leq 0.001$ per study day, Wald's test). More staff were involved in supine-to-prone positioning than prone-to-supine positioning on study day 1 (Signed rank test; $p=0.01$ ) but not on other days $(p \geq 0.31)$.

The length of time involved in the turning procedure was also longer in the prone group: 2 minutes (median; Q1-Q3, 1-5 minutes) for supine patient's side-to-side positioning, 7 minutes (median; Q1-Q3 3-10 minutes) for prone patient's supine-to-prone turn (compared to supine group: $p<0.05$ for days $1-5,7 ; p=0.08$ for day 6 ; Wilcoxon rank sum test), and 5 minutes (median; Q1-Q3, 3-10 minutes) for prone patient's prone-to-supine positioning (compared to supine group: $p \leq 0.01$ for days $1-5 ; p \geq 0.06$ for days $6-7$; Wilcoxon rank sum test). The time required to turn a patient from supine-to-prone was in general longer than the time required to return the patient supine ( $p<0.05$ for days 1,2,4,5,6; $p \geq 0.09$ for days 3 and 7 ; signed rank test).

\section{DISCUSSION}

Our data show that prone positioning can be accomplished safely in critically ill pediatric patients. We also demonstrate that these patients can be safely assessed and managed while maintained in the prone position for prolonged periods of time.

One of the most important concerns with prone positioning is the maintenance of an artificial airway during the repositioning maneuver and while the patient is maintained in the prone position. Marcano et al, (33) noted that prone positioning results in cephalad movement of ETT within the trachea. Our protocol incorporated their recommendation that the tip of the ETT be verified to be deeper than one third of the total length of the thoracic trachea before prone positioning. Unlike previous reports, $(8,34)$ no patient in the current study was inadvertently extubated during a repositioning cycle. Our inadvertent extubation rate was 0.85 per 100 ventilator days in the prone group. This rate is similar to the 0.72 to 1.26 rates previously reported in supine critically ill children $(35,36)$ and comparable to the 0.76 rate reported in adult prone positioned patients. (10) Therefore, our data demonstrate that, given proper care, there is not an increased incidence of inadvertent ETT extubation associated with turning or maintaining the prone position in pediatric patients. Proper care includes using a positioning protocol that assigns either a nurse or respiratory therapist the primary responsibility of maintaining the patient's airway during a turn and the avoidance of extreme head rotation when prone.

Two patients randomized to the prone group experienced an obstructed ETT during the study period. Many, including the first descriptive study on prone positioning,(37) anecdotally note that pulmonary drainage may increase in the prone position. Although not operationally defined, Gattinoni et al.(8) reported that $39 \%$ of their prone patients experienced an airway 
obstruction. Compared with supine patients, Guerin et al. (10) reported that the rate of ETT obstruction per 100 ventilator days was significantly greater in their prone group $(0.59$ [CI: $0.39-0.79$ ] prone vs. 0.23 [CI: $0.10-0.36$ ] supine). Our 0.42 rate of ETT obstruction per 100 ventilator days appears consistent with these adult data. Based on these findings, clinicians should remain vigilant for subtle signs indicating the need for ETT suctioning.

Several other safety concerns exist regarding the maintenance of adequate mechanical ventilatory support while in the prone position. These include disconnection of the ventilator circuit during a turn and potential changes in ETT leak when prone. In the current study, most of our patients were repositioned while connected to mechanical ventilation thus avoiding the loss of lung volume. There were also no significant differences in ETT leak between the two groups or within the prone group while supine or prone.

In the prone group, five patients managed on HFOV experienced significant hypercarbia. (9). One patient was withdrawn from all study protocols because of reproducible hypercarbia within several hours of prone positioning. One patient's hypercarbia was attributed to a kinked ETT after a head turn half-way through a 20-hour prone rotation. Upon follow-up, the tip of the patient's ETT was noted to be positioned high in the airway. Two patients were noted to be hypercarbic during the one-hour post prone turn blood gas. Clinically, the chest "wiggle" in these patients appeared diminished in the prone position suggesting a change in chest wall compliance. Both of these patients responded to an increase in HFOV power. The remaining patient with a tracheostomy was noted to be hypercarbic during the one-hour post supine blood gas. Clinically, the diminished chest "wiggle" in this patient was attributed to a mal-positioned tracheostomy tube. This patient responded to an airway adjustment. The increase in $\mathrm{PaCO}_{2}$ is particularly concerning for patients managed on $\mathrm{HFOV}$, since end-tidal $\mathrm{CO}_{2}$ cannot be monitored in this patient group. Therefore, assessment of blood gases following the turning procedure is crucial and the use of transcutaneous $\mathrm{CO}_{2}$ monitoring in small infants may be helpful.

In this study, we found that enteral feedings can be safely administered to patients in the prone position. No feeding complications, including pulmonary aspiration, necrotizing enterocolitis, or bowel perforation were reported. In addition, there were no significant differences between the supine and prone groups regarding their ability to reach full enteral calories. These findings are in contrast to previous reports where prone positioning was associated with higher rates of feeding complications, specifically, vomiting, increased gastric residuals, and inability to meet caloric goals. $(34,38,39)$ Critically ill pediatric patients in this study were positioned with their abdomen unrestrained from the bed and the head of bed elevated in a reverse trendelenburg position. In addition, most patients received transpyloric feedings.

We also confirmed previous reports that patients receiving transpyloric feedings reached their optimal calories sooner than those patients receiving gastric feedings.(40,41) Gastric emptying in critically ill patients can be delayed in this population and can lead to impaired tolerance of enteral feedings. (42) Unless contraindicated, our enteral feeding guideline specified the initiation of transpyloric feedings on study day one. Gastric feedings were used if preferred by the clinical team or if NJT placement was unsuccessful. Therefore, we cannot rule out a selection bias in patients fed by the gastric route because gastric paresis may have contributed to NJT placement failure.

Patient comfort in the prone position had not been systematically described. Pain scores during the acute phase of illness were low and most patients in both groups were responsive to touch or name. Even though bedside nurses were more likely to administer a preprocedural bolus of a comfort medication prior to a prone turn, the total amount of comfort medications received in the two groups was not significantly different. Previous pediatric studies report no 
increments in analgesia or sedation while patients were prone, $(1,6)$ while studies in adult patients report the need for increased sedation.(8)

Pressure ulcers are frequent complications associated with prone positioning. $(6,12)$ Our protocol, designed to maintain skin integrity, was based on what we learned during our phase one experience in which 8 Stage II pressure ulcers occurred in $24 \%$ of prone patients.(5) Unlike previous reports in adults, $(8,10,34)$ we observe fewer pressure ulcers in our prone positioned pediatric patients. The occurrence of pressure ulcers was similar between the two groups and similar to previous reports of pressure ulcers in critically ill children.(43) We believe that we were able to maintain skin integrity by adherence to a protocol which specified the use of pressure-relieving materials and patient repositioning every 2 hours. Based on our findings, prone positioning can be safely used as an additional turning surface to provide therapeutic and comfort management in critically ill children.

As with any new therapeutic intervention, staff resources required to successfully implement the strategy should be described. Although less than previous reports in adult patients, (8) more caregivers and more time were required to reposition pediatric patients to and from the prone position than required to adhere to the positioning protocols in this study. Safe prone positioning requires increased resources for short periods of time. In addition to nurses, other caregivers who can assist with this procedure include hospital-based lift teams and nursing care assistants.

In summary, prone positioning should continue to be used in critically ill children as a recruitment maneuver to improve oxygenation in patients with ALI and as a treatment strategy in patients with complex posterior wounds, bronchopulmonary compression from an anterior mediastinal mass, and anasarca. This is the first study clearly demonstrating that prone positioning can be safely accomplished in critically ill children. The potential for injury related to prone positioning is high considering that most clinicians learn to assess and manage critically ill patients while in the supine position. We showed that when a prone positioning protocol is used, prone positioning does not increase the risk of inadvertent endotracheal tube dislodgement, interruptions in mechanical ventilation, and pressure ulcers. In addition, nutrition and comfort management goals can be achieved while patients remain in the prone position for prolonged periods of time. As demonstrated by the significant mechanical ventilatory and circulatory support required by our patient sample, prone positioning can be accomplished even in the most critically ill patient. The implementation and adherence to a positioning protocol that prevents complications and evaluates patient response is essential to the successful use of prone positioning. Prone positioning offers a unique treatment option that should be considered in the management of critically ill pediatric patients.

\section{Supplementary Material}

Refer to Web version on PubMed Central for supplementary material.

\section{Acknowledgements}

The authors acknowledge the pediatric critical care nurses and respiratory therapists who participated in this clinical trial. Their vigilance helped us demonstrate the safety of prone positioning in a vulnerable patient group.

\section{References}

1. Kornecki A, Frndova H, Coates AL, Shemie SD. 4A randomized trial of prolonged prone positioning in children with acute respiratory failure. Chest 2001;119(1):211-218. [PubMed: 11157606]

2. Fridrich P, Krafft P, Hochleuthner H, Mauritz W. The effects of long-term prone positioning in patients with trauma-induced adult respiratory distress syndrome. Anesthesia \& Analgesia 1996;83(6):12061211. [PubMed: 8942587] 
3. Chatte G, Sab JM, Dubois JM, Sirodot M, Gaussorgues P, Robert D. Prone position in mechanically ventilated patients with severe acute respiratory failure. Am J Respir Crit Care Med 1997;155(2):473478. [PubMed: 9032181]

4. Pelosi P, Tubiolo D, Mascheroni D, Vicardi P, Crotti S, Valenza F, Gattinoni L. Effects of the prone position on respiratory mechanics and gas exchange during acute lung injury. Am J Respir Crit Care Med 1998;157(2):387-393. [PubMed: 9476848]

5. Curley MAQ, Thompson JE, Arnold JH. The effects of early and repeated prone positioning in pediatric patients with acute lung injury. Chest 2000;118(1):156-163. [PubMed: 10893373]

6. Casado-Flores J, MartÃ-nez de Azagra A, Ruiz-LÃ³ $\tilde{A}^{3}$ ez MJs, Ruiz M. Serrano A: Pediatric ARDS: effect of supine-prone postural changes on oxygenation. Intensive Care Med 2002;28(12):1792-1796. [PubMed: 12447525]

7. Relvas MS, Silver PC, Sagy M. Prone positioning of pediatric patients with ARDS results in improvement in oxygenation if maintained $>12$ h daily. Chest 2003;124(1):269-274. [PubMed: 12853533]

8. Gattinoni L, Tognoni G, Pesenti A, Taccone P, Mascheroni D, Labarta V, Malacrida R, Di Giulio P, Fumagalli R, Pelosi P, et al. Effect of prone positioning on the survival of patients with acute respiratory failure. N Engl J Med 2001;345(8):568-573. [PubMed: 11529210]

9. Curley MAQ, Hibberd PL, Fineman LD, Wypij D, Shih M-C, Thompson JE, Grant MJC, Barr FE, Cvijanovich NZ, Sorce L, et al. Effect of prone positioning on clinical outcomes in children with acute lung injury: a randomized controlled trial. JAMA 2005;294(2):229-237. [PubMed: 16014597]

10. Guerin C, Gaillard S, Lemasson S, Ayzac L, Girard R, Beuret P, Palmier B, Le QV, Sirodot M, Rosselli S, et al. Effects of Systematic Prone Positioning in Hypoxemic Acute Respiratory Failure: A Randomized Controlled Trial. JAMA 2004;292(19):2379-2387. [PubMed: 15547166]

11. Mancebo J, Rialp G, Fernandez R, Gordo F, Albert RK. Prone vs supine position in ARDS patients. Results of a randomized multicenter trial. Am J Respir Crit Care Med 2003;167(7):A180.

12. Curley MAQ. Prone positioning of patients with acute respiratory distress syndrome: a systematic review. Am J Crit Care 1999;8(6):397-405. [PubMed: 10553180]

13. Bernard GR, Artigas A, Brigham KL, Carlet J, Falke K, Hudson L, Lamy M, Legall JR, Morris A, Spragg R. The American-European Consensus Conference on ARDS. Definitions, mechanisms, relevant outcomes, and clinical trial coordination. Am J Respir Crit Care Med 1994;149(3 Pt 1):818824. [PubMed: 7509706]

14. Curley MAQ, Fackler JC. Weaning from mechanical ventilation: patterns in young children recovering from acute hypoxemic respiratory failure. Am J Crit Care 1998;7(5):335-345. [PubMed: 9740883]

15. Randolph AG, Wypij D, Venkataraman ST, Hanson JH, Gedeit RG, Meert KL, Luckett PM, Forbes $\mathrm{P}$, Lilley M, Thompson J, et al. Effect of mechanical ventilator weaning protocols on respiratory outcomes in infants and children: a randomized controlled trial. JAMA 2002;288(20):2561-1568. [PubMed: 12444863]

16. ARDSNet. Ventilation with lower tidal volumes as compared with traditional tidal volumes for acute lung injury and the acute respiratory distress syndrome. N Engl J Med 2000;342(18):1301-1308. [PubMed: 10793162]

17. Amato MB, Barbas CS, Medeiros DM, Magaldi RB, Schettino GP, Lorenzi-Filho G, Kairalla RA, Deheinzelin D, Munoz C, Oliveira R, et al. Effect of a protective-ventilation strategy on mortality in the acute respiratory distress syndrome.[see comment]. N Engl J Med 1998;338(6):347-354. [PubMed: 9449727]

18. Arnold JH, Hanson JH, Toro-Figuero LO, Gutierrez J, Berens RJ, Anglin DL. Prospective, randomized comparison of high-frequency oscillatory ventilation and conventional mechanical ventilation in pediatric respiratory failure. Crit Care Med 1994;22(10):1530-1539. [PubMed: 7924362]

19. Hickling KG, Henderson SJ, Jackson R. Low mortality associated with low volume pressure limited ventilation with permissive hypercapnia in severe adult respiratory distress syndrome. Intensive Care Med 1990;16(6):372-377. [PubMed: 2246418] 
20. Hickling KG, Walsh J, Henderson S, Jackson R. Low mortality rate in adult respiratory distress syndrome using low-volume, pressure-limited ventilation with permissive hypercapnia: a prospective study. Crit Care Med 1994;22(10):1568-1578. [PubMed: 7924367]

21. Drakulovic MB, Torres A, Bauer TT, Nicolas JM, Noguac S, Ferrer M. Supine body position as a risk factor for nosocomial pneumonia in mechanically ventilated patients: a randomised trial. Lancet 1999;354(9193):1851-1858. [PubMed: 10584721]

22. Curley MAQ, Dodson BL, Arnold JH. Designing a nurse-implemented sedation algorithm for use in a pediatric intensive care unit. Pediatr Crit Care Med 2003;4(3):A158.

23. Merkel SI, Voepel-Lewis T, Shayevitz JR, Malviya S. The FLACC: a behavioral scale for scoring postoperative pain in young children. Pediatr Nurs 1997;23(3):293-297. [PubMed: 9220806]

24. Solodiuk J, Curley MAQ. Pain assessment in nonverbal children with severe cognitive impairments: the Individualized Numeric Rating Scale (INRS). J Pediatr Nurs 2003;18(4):295-299. [PubMed: 12923744]

25. Oakes, L. Critical Care Nursing of Infants and Children. Second Edition edn. Philadelphia, PA: WB Saunders Co; 2001. Caring practices: Providing comfort; p. 558

26. Devlin JW, Boleski G, Mlynarek M, Nerenz DR, Peterson E, Jankowski M, Horst HM, Zarowitz BJ. Motor Activity Assessment Scale: a valid and reliable sedation scale for use with mechanically ventilated patients in an adult surgical intensive care unit. Crit Care Med 1999;27(7):1271-1275. [PubMed: 10446819]

27. Curley MAQ, Castillo L. Nutrition and shock in pediatric patients. New Horiz 1998;6(2):212-225. [PubMed: 9654328]

28. Harrison AM, Clay B, Grant MJ, Sanders SV, Webster HF, Reading JC, Dean JM, Witte MK. Nonradiographic assessment of enteral feeding tube position. Crit Care Med 1997;25(12):20552059. [PubMed: 9403759]

29. Bergstrom, NAR.; Alvarez, OM., et al. Treatment of Pressure Ulcers. Clinical Practice Guideline. No. 15. Rockville, MD: 1994.

30. Curley MA, Quigley SM, Lin M. Pressure ulcers in pediatric intensive care: incidence and associated factors. Pediatr Crit Care Med 2003;4(3):284-290. [PubMed: 12831408]

31. Panel NPUA. Pressure ulcers prevalence, cost and risk assessment: consensus development conference statement. Decubitus 1989;2(2):24-28.

32. Stage I Assessment in Darkly Pigmented Skin. http://www.npuap.org/positn4.html

33. Marcano BV, Silver P, Sagy M. Cephalad movement of endotracheal tubes caused by prone positioning pediatric patients with acute respiratory distress syndrome. Pediatr Crit Care Med 2003;4 (2):186-189. [PubMed: 12749650]

34. L'Her E, Renault A, Oger E, Robaux M-A, Boles J-M. A prospective survey of early 12-h prone positioning effects in patients with the acute respiratory distress syndrome. Intensive Care Med 2002;28(5):570-575. [PubMed: 12029404]

35. Little LA, Koenig JC Jr, Newth CJ. Factors affecting accidental extubations in neonatal and pediatric intensive care patients. Crit Care Med 1990;18(2):163-165. [PubMed: 2298007]

36. Rivera R, Tibballs J. Complications of endotracheal intubation and mechanical ventilation in infants and children. Crit Care Med 1992;20(2):193-199. [PubMed: 1737455]

37. Piehl MA, Brown RS. Use of extreme position changes in acute respiratory failure. Crit Care Med 1976;4(1):13-14. [PubMed: 1253612]

38. Reignier J, Thenoz-Jost N, Fiancette M, Legendre E, Lebert C, Bontemps F, Clementi E, MartinLefevre L. Early enteral nutrition in mechanically ventilated patients in the prone position. Crit Care Med 2004;32(1):94-99. [PubMed: 14707565]

39. Blanch L, Mancebo J, Perez M, Martinez M, Mas A, Betbese AJ, Joseph D, Ballus J, Lucangelo U, Bak E. Short-term effects of prone position in critically ill patients with acute respiratory distress syndrome. Intensive Care Med 1997;23(10):1033-1039. [PubMed: 9407238]

40. Meert KL, Daphtary KM, Metheny NA. Gastric vs small-bowel feeding in critically ill children receiving mechanical ventilation: a randomized controlled trial. Chest 2004;126(3):872-878. [PubMed: 15364769] 
41. Heyland DK, Drover JW, Dhaliwal R, Greenwood J. Optimizing the benefits and minimizing the risks of enteral nutrition in the critically ill: role of small bowel feeding. JPEN J Parenter Enteral Nutr 2002;26(6 Suppl):S51. [PubMed: 12405623]

42. Heyland DK, Tougas G, King D, Cook DJ. Impaired gastric emptying in mechanically ventilated, critically ill patients. Intensive Care Med 1996;22(12):1339-1344. [PubMed: 8986483]

43. Curley MAQ, Quigley SM, Lin M. Pressure ulcers in pediatric intensive care: incidence and associated factors. Pediatr Crit Care Med 2003;4(3):284-290. [PubMed: 12831408]

44. Pollack MM, Patel KM, Ruttimann UE. The pediatric risk of mortality III-- Acute physiology score (PRISM III-APS): A method of assessing physiologic instability for pediatric intensive care unit patients. J Pediatr 1997;131(4):575-581. [PubMed: 9386662]

45. Gattinoni L, Pelosi P, Suter PM, Pedoto A, Vercesi P, Lissoni A. Acute respiratory distress syndrome caused by pulmonary and extrapulmonary disease. Different syndromes? Am J Respir Crit Care Med 1998;158(1):3-11. [PubMed: 9655699] 
Table 1

Patient Characteristics at Enrollment*

(N=102)

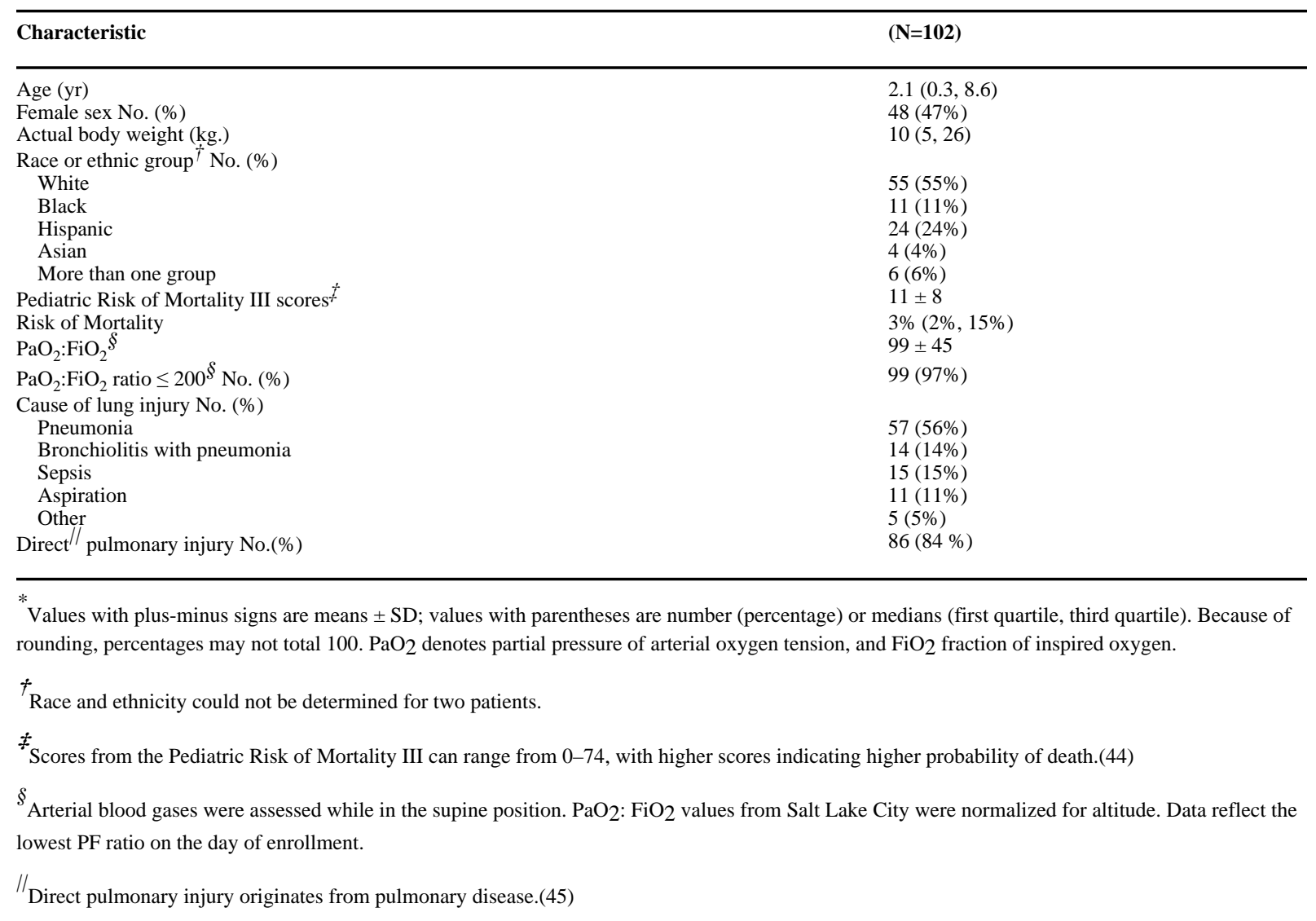


Table 2

Acute Phase Changes in Ventilator Settings Related to Repositioning in the Prone Group*

\begin{tabular}{|c|c|c|c|}
\hline & $\begin{array}{l}\text { Mean } \pm \text { SD over the } \\
\text { acute phase }\end{array}$ & Pre and Post Supine-Prone Turn & $\begin{array}{l}\text { Pre and Post Prone-Supine } \\
\text { Turn }\end{array}$ \\
\hline \multicolumn{4}{|l|}{ Conventional Mechanical Ventilation } \\
\hline $\mathrm{FiO}_{2}$ & $0.48 \pm 0.15$ & $\begin{array}{l}\mathrm{N}=128 \\
-0.02(-0.03,-0.00)^{\dagger}\end{array}$ & $\begin{array}{l}\mathrm{N}=124 \\
0.01(-0.00,0.03)\end{array}$ \\
\hline Exhaled tidal volume (mL.) & $130 \pm 113$ & $\begin{array}{l}\mathrm{N}=123 \\
-0.3(-2.9,2.3)\end{array}$ & $\begin{array}{l}\mathrm{N}=122 \\
2.5(-4.6,9.6)\end{array}$ \\
\hline $\begin{array}{l}\text { Set ventilator rate (breaths per } \\
\text { minute) }\end{array}$ & $23 \pm 9$ & $\begin{array}{l}\mathrm{N}=128 \\
0.0(-0.2,0.2)\end{array}$ & $\begin{array}{l}\mathrm{N}=125 \\
-0.2(-0.4,0.0)\end{array}$ \\
\hline Set PIP $\left(\mathrm{cmH}_{2} \mathrm{O}\right)$ & $25 \pm 6$ & $\begin{array}{l}\mathrm{N}=57 \\
-0.3(-0.8,0.1)\end{array}$ & $\begin{array}{l}\mathrm{N}=57 \\
0.0(-0.2,0.2)\end{array}$ \\
\hline Set PEEP $\left(\mathrm{cmH}_{2} \mathrm{O}\right)$ & $8 \pm 3$ & $\begin{array}{l}\mathrm{N}=128 \\
-0.1(-0.1,0.0)\end{array}$ & $\begin{array}{l}\mathrm{N}=125 \\
-0.0(-0.1,0.1)\end{array}$ \\
\hline Mean airway pressure $\left(\mathrm{cmH}_{2} \mathrm{O}\right)$ & $14 \pm 4$ & $\begin{array}{l}\mathrm{N}=128 \\
-0.3(-0.5,0.0)\end{array}$ & $\begin{array}{l}\mathrm{N}=124 \\
-0.3(-0.6,-0.0)^{*}\end{array}$ \\
\hline Pressure support $\left(\mathrm{cmH}_{2} \mathrm{O}\right)$ & $10 \pm 4$ & $\begin{array}{l}\mathrm{N}=104 \\
100 \% \text { unchanged }\end{array}$ & $\begin{array}{l}\mathrm{N}=108 \\
-0.1(-0.2,0.0)\end{array}$ \\
\hline I:E ratio (set) & $0.57 \pm 0.26$ & $\begin{array}{l}\mathrm{N}=128 \\
-0.01(-0.03,0.02)\end{array}$ & $\begin{array}{l}\mathrm{N}=126 \\
-0.02(-0.05,0.01)\end{array}$ \\
\hline Plateau pressure (VC mode) & $30 \pm 8$ & $\begin{array}{l}\mathrm{N}=190.0 \\
(-1.4,1.4)\end{array}$ & $\begin{array}{l}\mathrm{N}=14 \\
-1.6(-3.0,-0.3)\end{array}$ \\
\hline OSCILLATOR (HFOV) SETTINGS & & & \\
\hline $\mathrm{FiO}_{2}$ & $0.59 \pm 0.15$ & $\begin{array}{l}\mathrm{N}=71 \\
-0.00(-0.01,0.01)\end{array}$ & $\begin{array}{l}\mathrm{N}=67 \\
0.06(0.02,0.11) \S\end{array}$ \\
\hline Hertz (Hz) & $7.5 \pm 2.9$ & $\begin{array}{l}\mathrm{N}=71 \\
100 \% \text { no change }\end{array}$ & $\begin{array}{l}\mathrm{N}=67 \\
-0.01(-0.04,0.01)\end{array}$ \\
\hline Mean Airway Pressure $\left(\mathrm{cmH}_{2} \mathrm{O}\right)$ & $24 \pm 5$ & $\begin{array}{l}\mathrm{N}=71 \\
-0.1(-0.3,0.1)\end{array}$ & $\begin{array}{l}\mathrm{N}=67 \\
0.2(-0.1,0.6)\end{array}$ \\
\hline Delta P & $50 \pm 17$ & $\begin{array}{l}\mathrm{N}=71 \\
-0.1(-0.5,0.3)\end{array}$ & $\begin{array}{l}\mathrm{N}=67 \\
0.1(-0.8,1.0)\end{array}$ \\
\hline$\%$ Inspiratory Time & $33 \pm 0.2$ & $\begin{array}{l}N=71 \\
100 \% \text { unchanged }\end{array}$ & $\begin{array}{l}\mathrm{N}=67 \\
0.01(-0.01,0.04)\end{array}$ \\
\hline
\end{tabular}

* mean change (95\% CI); N = Number of turn cycles; (Note. There were a total of 202 supine-prone-supine cycles. For the supine-to-prone turns, we describe 128 turns on conventional mechanical ventilation and 71 on HFOV. For prone-to-supine turns, we describe 126 turns on conventional mechanical ventilation and 69 on HFOV. Turns excluded from analyses include those with missing data or those in which the mode of ventilation changed pre and post turn.).

${ }_{p}^{+}=0.01$

$\stackrel{\neq}{*}=0.02$

$\S_{p=0.005}$ 


\section{Table 3}

Location of pressure ulcers

\begin{tabular}{llll}
\hline & Total group (N=26) & Prone (N= 13) & Supine (N=13) \\
\hline Shin/ankle & $1(4 \%)$ & $1(8 \%)$ & 0 \\
Chest & $4(15 \%)$ & $4(31 \%)$ & 0 \\
Shoulder & $2(8 \%)$ & $1(8 \%)$ & $1(8 \%)$ \\
Heel & $1(4 \%)$ & $1(8 \%)$ & 0 \\
Coccyx & $4(15 \%)$ & 0 & $4(31 \%)$ \\
Vertebral & $1(4 \%)$ & 0 & $1(8 \%)$ \\
Scapular & $1(4 \%)$ & 0 & $1(8 \%)$ \\
Ear & $5(19 \%)$ & $2(15 \%)$ & $3(23 \%)$ \\
Chin & $4(15 \%)$ & 0 & 0 \\
Occiput & $3(12 \%)$ & $0(31 \%)$ & $3(23 \%)$ \\
\end{tabular}

